\title{
Role of Blood Stasis Syndrome of Kampo Medicine in the Early Pathogenic Stage of Atherosclerosis: A Retrospective Cross-Sectional Study
}

\author{
Akira Morita $\mathbb{D}^{1},{ }^{1}$ Takao Namiki, ${ }^{1}$ Toshiya Nakaguchi, ${ }^{2}$ Kazunari Murai, ${ }^{3}$ Yuki Watanabe, ${ }^{1}$ \\ Michimi Nakamura, ${ }^{1}$ Yohei Kawasaki, ${ }^{4}$ Yuki Shiko, ${ }^{4}$ Yutaka Tamura, ${ }^{5}$ Akiko Suganami, ${ }^{5}$ \\ Aya Murakami, ${ }^{6}$ Akio Yagi, ${ }^{1}$ Hideki Okamoto, ${ }^{1}$ and Yoshiro Hirasaki ${ }^{1}$ \\ ${ }^{1}$ Department of Japanese-Oriental (Kampo) Medicine, Graduate School of Medicine, Chiba University, 1-8-1 Inohana, Chuo-ku, \\ Chiba 260-8670, Japan \\ ${ }^{2}$ Center for Frontier Medical Engineering, Chiba University, 1-33, Yayoi-cho, Inage-ku, Chiba 263-8522, Japan \\ ${ }^{3}$ Graduate School of Engineering, Chiba University, 1-33, Yayoi-cho, Inage-ku, Chiba 263-8522, Japan \\ ${ }^{4}$ Biostatistics Section, Clinical Research Center, Chiba University Hospital, 1-8-1 Inohana, Chuo-ku, Chiba 260-8677, Japan \\ ${ }^{5}$ Department of Bioinformatics, Graduate School of Medicine, Chiba University, 1-8-1 Inohana, Chuo-ku, Chiba 260-8670, Japan \\ ${ }^{6}$ Center for Pharmaceutical Education, Faculty of Pharmacy, Yokohama University of Pharmacy, 601 Matano-cho, Totsuka-ku, \\ Yokohama 245-0066, Japan
}

Correspondence should be addressed to Akira Morita; amorita@chiba-u.jp

Received 28 January 2021; Revised 15 March 2021; Accepted 26 April 2021; Published 27 May 2021

Academic Editor: Dan Su

Copyright (C) 2021 Akira Morita et al. This is an open access article distributed under the Creative Commons Attribution License, which permits unrestricted use, distribution, and reproduction in any medium, provided the original work is properly cited.

In Kampo medicine, blood stasis (BS) syndrome is strongly associated with microangiopathy and can lead to atherosclerosis. Vascular endothelial dysfunction (VED), evaluated through flow-mediated dilation (FMD), plays an important role in the early stages of atherosclerosis. However, the association of BS syndrome with VED, as determined using FMD, has not been reported. This study investigated the association between BS syndrome and VED using FMD. Forty-one patients with normal glucose tolerance or impaired glucose tolerance (IGT) and without macrovascular complications were evaluated using FMD from May 2017 to August 2017. Based on the BS score, the patients were divided into the non-BS $(n=19)$ and BS syndrome $(n=22)$ groups. Physical and background characteristics, physiological function test results, and laboratory data were compared. Univariate analysis revealed that FMD and a history of dyslipidemia/IGT were significantly different between the two groups $(p<0.05)$. Multiple logistic regression analysis showed that BS syndrome was significantly associated with FMD (odds ratio: $6.26 ; p=0.03$ ) after adjusting for the history of dyslipidemia/IGT. The receiver operating characteristic curve showed that the area under the curve for BS syndrome $(0.74 ; p<0.001)$ and history of IGT $(p<0.007)$ provided good diagnostic accuracy for FMD. The area under the curve for "BS syndrome + IGT" showed very good accuracy $(0.80 ; p<0.0001)$ and was higher than that for BS syndrome or IGT alone. In conclusion, the results of this study suggest that the BS score in Kampo medicine could be a useful tool for detecting the early pathogenic stages of atherosclerosis.

\section{Introduction}

Various pathological conditions can cause vascular endothelial dysfunction (VED) in the first stage of atherosclerotic changes. These pathological conditions are often related to lifestyle behaviors, such as an unbalanced diet, lack of sleep or exercise, excessive drinking, stress, and smoking. Moreover, they are recognized as life-threatening owing to the associated risks of cardiovascular and cerebrovascular disease $[1,2]$.

According to Kampo medicine theory, the human body is composed of three major elements: qi, blood, and fluid. 
Abnormalities in these elements help define Mibyou, the socalled "disease-oriented" healthy stage [3] which can cause subjective symptoms in patients; however, this condition is often not detected by Western medicine due to the absence of abnormal results in routine tests such as blood tests or physiological function tests. In particular, abnormalities in the blood element affect blood stasis (BS), an important pathophysiologic concept that was first documented in the huangdi neijing - a medical text that served as a foundation for two other documents, the shanghan lun and jin gui yao lue, and as a subject of many research studies. Based on ancient Chinese medical texts, BS is described as a blood circulation disorder that causes various symptoms, including actual BS, reduced blood flow, and cessation of blood flow [4]. BS is termed yu xue in Chinese, eohyul in Korean, and oketsu in Japanese [5].

Efforts toward a scientific approach in the diagnosis of BS have been unsuccessful, partly owing to the heterogeneity in its assessment by practitioners with varying experience. Therefore, Terasawa et al. advocated the use of the BS score as the basis for the diagnostic criteria of BS (Table 1) [6]. The BS score is based on traditional East Asian medicine text, as well as on previous research. Its data have been analyzed statistically; thus, the BS score is considered as a quantitative standard and is commonly used in various clinical studies on BS syndrome [3-12].

In this study, patients with a total score of less than 21 were classified into the non-blood stasis group, while those with a total score greater than or equal to 21 were classified into the blood stasis syndrome group. Mild symptoms were designated by half points.

BS syndrome is assumed to be associated with microangiopathy $[7,8]$, which in turn can lead to atherosclerosis [13]. In recent animal studies, the velocity of the microcirculation increased with the administration of keishibukuryogan (Gui-zhi-fu-ling-wan in Chinese), a Kampo formulation that acts as a BS-ameliorating agent [14].

The ankle-brachial index (ABI) is used mainly for the diagnosis of morphological changes caused by vascular blockage. This index was developed in the mid-1990s, using an oscillometric method [15]. The cardioankle vascular index (CAVI) is a measure of the elastic force of the entire artery, from the origin of the aorta to the ankle. It is used to calibrate the elasticity of a blood vessel to blood pressure using the stiffness parameter $\beta$, which indicates vascular hardness [15].

VED, which can be evaluated using flow-mediated dilation (FMD), is an early functional change of atherosclerosis. It leads to arterial stiffness, which can be assessed using CAVI, and causes arterial stenosis, which can be evaluated using the ABI. Eventually, this process can result in cardiovascular and cerebrovascular diseases [1, 2]. VED plays a crucial role in the early pathogenic stages of atherosclerosis [16]. Conventional risk factors, such as smoking [17], hypertension [18], dyslipidemia (DLP) [19], and diabetes mellitus [20], have been associated with the severity of VED, and VED has been reported to be an independent risk factor for atherosclerosis [1, 21, 22]. VED has also been associated with microangiopathy in patients with diabetes mellitus [23].
FMD was developed to evaluate the brachial endothelial function and is now widely used as a surrogate marker in clinical trials [24, 25]. FMD is used to study VED using a simple, noninvasive medical ultrasound apparatus designed to measure the change in the forearm vascular diameter caused by the production of nitric oxide. An association of BS syndrome with VED, using FMD, has not been previously reported.

In this study, we investigated the relationship between the BS syndrome of Kampo medicine and VED, as determined by FMD, by examining various parameters including physical and background characteristics, physiological function test results, and laboratory test results of patients who underwent screening for atherosclerosis (Tables S1-S4 in the Supplementary Material).

\section{Materials and Methods}

2.1. Patients. This retrospective, cross-sectional study included 41 consecutive patients (17 men and 24 women; average age $70.1 \pm 11.9$ years; range $33-83$ years) with either normal glucose tolerance or impaired glucose tolerance (IGT) and who had no macrovascular complications. Patients were screened for atherosclerosis at the Department of Japanese-Oriental (Kampo) Medicine at Chiba University Hospital between May and August 2017. Twenty-one of the 24 patients in this study were postmenopausal (Figure 1).

2.2. Ethical Approval. The study protocol was approved by the Chiba University Hospital Certified Clinical Research Review Board (registration number 1932) on October 6, 2014. Patients provided written informed consent. All processes were conducted in accordance with the tenets of the Declaration of Helsinki (1964).

2.3. Classification of the BS Score. Five practitioners of Kampo medicine, with $\geq 10$ years of experience in clinical practice [26], classified the 41 patients into the non-BS (NBS) group and BS syndrome group based on the BS score. This diagnostic procedure was conducted as per standard clinical practice. The BS score contains 17 evaluation items, with total scores ranging from 0 to 90 points for men and from 0 to 101 points for women. Regardless of sex, patients with total scores $<21$ were classified into the NBS group and those with total scores $\geq 21$ were classified into the BS syndrome group (Table 1).

2.4. Physical and Background Characteristics. Physical characteristics obtained from the patients' medical records included age, sex, height, and body weight; the two latter characteristics were used to calculate the body mass index $\left(\mathrm{BMI}=\right.$ body weight $($ in $\mathrm{kg}) /$ height $^{2}$ (in meters)). Data on systolic blood pressure (SBP), diastolic blood pressure (DBP), and background characteristics such as smoking history and past medical history were also collected. 
TABLE 1: Diagnostic criteria for blood stasis syndrome.

\begin{tabular}{lcc}
\hline Symptom & Males & Score \\
\hline Dark-rimmed eyes & 10 & Females \\
Areas of dark pigmentation of facial skin & 2 & 10 \\
Rough skin & 2 & 2 \\
Livid lips & 10 & 5 \\
Livid gingiva & 10 & 5 \\
Livid tongue & 2 & 5 \\
Telangiectasis/vascular spiders & 2 & 10 \\
Subcutaneous hemorrhage & 5 & 10 \\
Palmar erythema & 10 \\
Resistance and tenderness on pressure of the left paraumbilical region & 5 & 5 \\
Resistance and tenderness on pressure of the right paraumbilical region & 5 & 5 \\
Resistance and tenderness on pressure of the umbilical region & 5 \\
Resistance and/or tenderness on pressure of the ileocecal region & 5 & 10 \\
Resistance and/or tenderness on pressure of the sigmoidal region & 10 \\
Resistance and/or tenderness on pressure of the subcostal region & - & 5 \\
Hemorrhoids & 90 \\
Dysmenorrhea & 5 \\
Total score & 5 \\
\hline
\end{tabular}

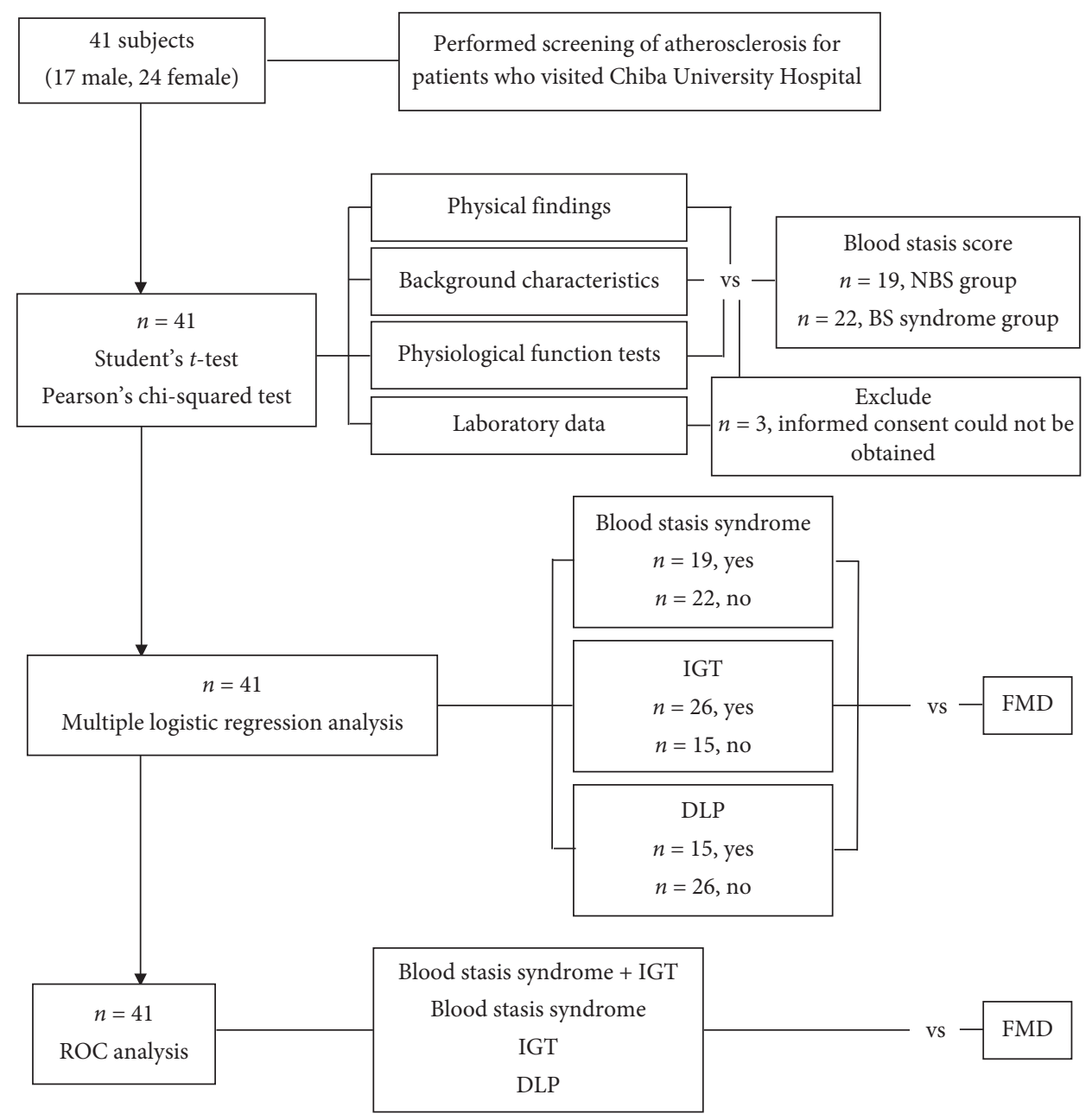

FIgURE 1: Participant selection. The patients were divided into the NBS group and BS syndrome group. Three patients were excluded from laboratory data analysis because we could not obtain informed consent. The variables found to be associated with the BS score were further tested by multiple logistic regression analysis to investigate the independent factors associated with FMD. To assess the ability of each variable to discriminate FMD, the areas under the ROC curves were calculated. NBS: non-blood stasis; BS: blood stasis; IGT: impaired glucose tolerance; DLP: dyslipidemia; FMD: flow-mediated dilation; ROC; receiver operating characteristic. 


\section{FMD}

Ultrasound studies to evaluate endothelial function were conducted from approximately 8:00 AM to 12:00 PM in a temperature-controlled room $\left(22-26^{\circ} \mathrm{C}\right)$, with the patient in a fasting, resting, and supine position state. Meals and all types of beverages, except water, were prohibited from the night before the study. The diameter of the patient's brachial artery was measured according to the Japan Circulation Society (JCS) guidelines for noninvasive vascular function tests (JCS 2013) [15]. A semiautomatic ultrasound device (UNEXEF 38 G; UNEX, Nagoya, Japan) was used to determine the vasodilator responses of the brachial artery.

Avascularization was performed by inflating a blood pressure cuff to $\mathrm{SBP}+50 \mathrm{~mm} \mathrm{Hg}$ for $5 \mathrm{~min}$. An arterial image was obtained at approximately $1 \mathrm{~min}$ after deflation. The FMD was quantified as the percentage change in brachial artery diameter within $1 \mathrm{~min}$ after avascularization, relative to the baseline vessel diameter. As in previous studies, the cutoff value of FMD was set at 5\% [27, 28]. The VED was constantly evaluated by monitoring the FMD.

3.1. Physiological Function Tests for Atherosclerosis. Physiological function tests (i.e., ABI and CAVI) were performed $10 \mathrm{~min}$ after measuring the FMD, according to the guidelines for noninvasive vascular function testing [15]. We added the right and left measured values and calculated the average value to determine the ABI and CAVI values (VaSera VS-1500N; Fukuda Denshi, Tokyo, Japan).

3.2. Laboratory Data. Informed consent could not be obtained from three patients; therefore, blood tests were conducted on 38 out of the 41 patients at the hospital's clinical laboratory department on the same day after the physiological function testing. We used the clinical laboratory data for triglycerides, high-density lipoprotein cholesterol, low-density lipoprotein cholesterol, hemoglobin A1c, fasting plasma glucose (FPG), and immunoreactive insulin (IRI). The latter two were used to calculate the homeostasis model assessment ratio (HOMA-R):

$$
\text { HOMA-R }=\text { IRI }\left[\frac{\mu U}{\mathrm{~mL}}\right] \times \mathrm{FPG} \frac{[\mathrm{mg} / \mathrm{dL}]}{405},
$$

and homeostasis model assessment beta-cell function (HOMA- $\beta$ ):

$$
\text { HOMA }-\beta=\text { FPG }\left[\frac{\mathrm{mg}}{\mathrm{dL}}\right] \times \frac{360}{\mathrm{IRI}}\left[\frac{\mu \mathrm{U}}{\mathrm{mL}}\right]-63,
$$

which are used to evaluate whether the patient had normal glucose tolerance or IGT. High-sensitivity C-reactive protein (hsCRP) levels were measured by standard laboratory techniques at our hospital and at the Special Reference Laboratories (Tokyo, Japan).

3.3. Statistical Analysis. The results are expressed as the number and percentage of cases for discrete variables and the mean \pm standard deviation for continuous variables. For assessment of patient characteristics, Student's $t$-test was applied when the variables were continuous, and Pearson's chi-squared test was used when the variables were discrete.

The variables found to be associated with the BS score were further tested by multiple logistic regression analysis to investigate the independent factors associated with FMD. The multiple logistic regression analysis was also used to determine the odds ratios (ORs) and the 95\% confidence intervals for BS syndrome as a factor associated with FMD compared with NBS.

To assess the ability of each variable to discriminate FMD, the areas under the receiver operating characteristic (ROC) curves (AUCs) were calculated. In addition to the asymptotic $95 \%$ confidence interval, $p$ values under the null hypothesis (true area $=0.50$ ) were calculated. An AUC of $>0.9$ was considered excellent; $0.8-0.9$, very good; $0.7-0.8$, good; $0.6-0.7$, average; and $<0.6$, poor [29, 30]. The significance level was set at $<5 \%$ in each analysis. All statistical analyses of the recorded data were performed using the Excel statistical software package (Statcel 4; OMS Publishing Inc., Saitama, Japan) and SAS statistical software package, version 9.4 (SAS Institute, Cary, NC, USA).

\section{Results}

4.1. Patient Characteristics. Baseline characteristics of the patients in the NBS and BS syndrome groups who completed all the tests are summarized in Table 2. The BS syndrome group had a significantly lower FMD $(4.9 \pm 1.4$ vs. $3.3 \pm 1.7$, $p<0.01)$ than the NBS group; moreover, the BS syndrome group had a significantly higher prevalence of a history of DLP/IGT $(p<0.05 / p<0.01)$ than the NBS group. Other variables were not significantly different between the groups.

4.2. Laboratory Data. Laboratory data of 38 patients out of the entire study population are summarized by group in Table 3. No significant differences were found between the groups.

4.3. Factors Associated with FMD. A multiple logistic regression analysis showed that BS syndrome was significantly associated with FMD (OR: 6.26; $p=0.03$ ) after adjusting for a history of DLP and IGT, both of which were not significant in the model (Table 4). The OR indicates that BS syndrome has a greater risk than NBS.

ROC curves were generated using BS syndrome, DLP, and IGT to determine whether they could distinguish NBS patients from BS syndrome patients. BS syndrome and IGT showed modest utility, with the ROC curves being higher and shifted to the left compared to DLP, which had poor fitting and utility. The AUCs of BS syndrome and IGT individually showed a good fit ( 0.74 and 0.71 , respectively) and were not significantly different from each other. However, the AUC for DLP was 0.62, which failed to reject the null hypothesis (true area $=0.50$ ). We performed multiple logistic regression to identify a better discriminator of FMD. We set the model using FMD as a dependent variable and the combination of BS syndrome and IGT as a single 
TABLE 2: Patient characteristics of the NBS group and BS syndrome group $(n=41)$.

\begin{tabular}{|c|c|c|c|c|}
\hline Variables & Units & NBS group $(n=19)$ & BS syndrome group $(n=22)$ & $p$ value \\
\hline Age & Years & $64.2 \pm 12.9$ & $70.9 \pm 10.6$ & $0.06^{\mathrm{a}}$ \\
\hline Sex & Male/female & $5 / 14$ & $12 / 10$ & $0.07^{\mathrm{b}}$ \\
\hline BMI & $\mathrm{kg} / \mathrm{m}^{2}$ & $22.6 \pm 3.8$ & $24.4 \pm 3.5$ & $0.13^{\mathrm{a}}$ \\
\hline SBP & $\mathrm{mmHg}$ & $128.4 \pm 19.3$ & $134.5 \pm 19.3$ & $0.33^{\mathrm{a}}$ \\
\hline DBP & $\mathrm{mmHg}$ & $74.3 \pm 8.8$ & $70.4 \pm 13.6$ & $0.30^{\mathrm{a}}$ \\
\hline Smoking & Yes/no & $5 / 14$ & $9 / 13$ & $0.33^{\mathrm{b}}$ \\
\hline Hypertension & Yes/no & $8 / 11$ & $13 / 9$ & $0.28^{\mathrm{b}}$ \\
\hline DLP & Yes/no & $3 / 16$ & $12 / 10$ & $<0.05^{\mathrm{b}}$ \\
\hline Cardiovascular disease & Yes/no & $1 / 18$ & $3 / 19$ & $0.37^{\mathrm{b}}$ \\
\hline Cerebrovascular disease & Yes/no & $3 / 16$ & $4 / 18$ & $0.84^{\mathrm{b}}$ \\
\hline IGT & Yes/no & $8 / 11$ & $18 / 4$ & $<0.01^{\mathrm{b}}$ \\
\hline \multicolumn{5}{|l|}{ Diabetic complications } \\
\hline Neuropathy & Yes/no & $1 / 18$ & $2 / 20$ & $0.64^{\mathrm{b}}$ \\
\hline Retinopathy & Yes/no & $0 / 19$ & $2 / 20$ & $0.18^{\mathrm{b}}$ \\
\hline Nephropathy & Yes/no & $0 / 19$ & $2 / 20$ & $0.18^{\mathrm{b}}$ \\
\hline Antithrombotic drug & Yes/no & $8 / 11$ & $13 / 9$ & $0.28^{\mathrm{b}}$ \\
\hline $\mathrm{ABI}$ & & $1.10 \pm 0.08$ & $1.04 \pm 0.11$ & $0.07^{\mathrm{a}}$ \\
\hline CAVI & & $8.4 \pm 1.5$ & $9.2 \pm 1.2$ & $0.07^{\mathrm{a}}$ \\
\hline FMD & $\%$ & $4.9 \pm 1.4$ & $3.3 \pm 1.7$ & $<0.01^{\mathrm{a}}$ \\
\hline
\end{tabular}

NBS: non-blood stasis; BS: blood stasis; BMI: body mass index; SBP: systolic blood pressure; DBP: diastolic blood pressure; DLP: dyslipidemia; IGT: impaired glucose tolerance; ABI: ankle-brachial index; CAVI: cardioankle vascular index; FMD: flow-mediated dilation. ${ }^{\mathrm{a}}$ Comparison between the groups by Student's $t$-test. ${ }^{\mathrm{b}}$ Comparison between the groups by Pearson's chi-squared test. Data are expressed as the mean \pm standard deviation.

TABLE 3: Blood test data of the NBS group and BS syndrome group $(n=38)$.

\begin{tabular}{|c|c|c|c|c|}
\hline Variables & Units & NBS group $(n=18)$ & BS syndrome group $(n=20)$ & $p$ value \\
\hline TG & $\mathrm{mg} / \mathrm{dL}$ & $114.4 \pm 63.0$ & $123.4 \pm 61.4$ & $0.67^{\mathrm{a}}$ \\
\hline HDL cholesterol & $\mathrm{mg} / \mathrm{dL}$ & $65.6 \pm 21.0$ & $56.7 \pm 11.3$ & $0.12^{\mathrm{a}}$ \\
\hline LDL cholesterol & $\mathrm{mg} / \mathrm{dL}$ & $122.8 \pm 41.1$ & $118.9 \pm 31.7$ & $0.75^{\mathrm{a}}$ \\
\hline $\mathrm{HbA} 1 \mathrm{c}$ & NGSP, \% & $6.2 \pm 0.9$ & $6.6 \pm 0.8$ & $0.21^{\mathrm{a}}$ \\
\hline FPG & $\mathrm{mg} / \mathrm{dL}$ & $113.9 \pm 24.6$ & $125.9 \pm 31.6$ & $0.22^{\mathrm{a}}$ \\
\hline IRI & $\mu \mathrm{U} / \mathrm{mL}$ & $4.3 \pm 2.4$ & $8.8 \pm 10.0$ & $0.08^{\mathrm{a}}$ \\
\hline HOMA-R & & $1.3 \pm 0.8$ & $3.4 \pm 5.4$ & $0.11^{\mathrm{a}}$ \\
\hline HOMA- $\beta$ & $\%$ & $34.5 \pm 26.2$ & $42.3 \pm 29.7$ & $0.41^{\mathrm{a}}$ \\
\hline hsCRP & $\mathrm{mg} / \mathrm{dL}$ & $0.11 \pm 0.18$ & $0.31 \pm 0.52$ & $0.13^{\mathrm{a}}$ \\
\hline
\end{tabular}

NBS: non-blood stasis; BS: blood stasis; TG: triglyceride; HDL: high-density lipoprotein; LDL: low-density lipoprotein; HbA1c: hemoglobin A1c; FPG: fasting plasma glucose; IRI: immunoreactive insulin; HOMA-R: homeostasis model assessment ration; HOMA- $\beta$ : homeostasis model assessment of beta-cell

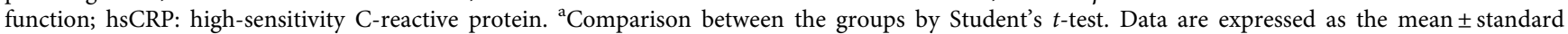
deviation.

TABLE 4: Multiple logistic regression analysis for FMD as a dependent variable and BS syndrome, IGT, and DLP as independent variables $(n=41)$.

\begin{tabular}{|c|c|c|c|c|}
\hline Variables & & OR & $95 \% \mathrm{CI}$ & $p$ value \\
\hline \multirow{2}{*}{ BS syndrome } & $\geq 21$ & 6.26 & $1.17-33.52$ & 0.03 \\
\hline & $<21$ & Reference & & \\
\hline \multirow{2}{*}{ IGT } & Yes & 4.12 & $0.73-23.06$ & 0.11 \\
\hline & No & Reference & & \\
\hline \multirow{2}{*}{ DLP } & Yes & 0.75 & $0.10-5.49$ & 0.78 \\
\hline & No & Reference & & \\
\hline
\end{tabular}

FMD: flow-mediation dilation; BS: blood stasis; IGT: impaired glucose tolerance; DLP: dyslipidemia; OR: odds ratio; CI: confidence interval. $\mathrm{FMD}<5 \%$.

independent variable ("BS syndrome + IGT"). The AUC of the combined "BS syndrome + IGT" parameter (0.80) showed a better fit than that of either BS syndrome or IGT (Table 5 and Figure 2).

\section{Discussion}

In this study, ROC analysis indicated that the AUCs of BS syndrome and IGT showed a fair diagnostic accuracy for FMD. Furthermore, the AUC of the combined "BS syndrome $+\mathrm{IGT}^{\text {" }}$ parameter showed better diagnostic accuracy than that of BS syndrome or IGT. The AUCs of BS syndrome and IGT were 0.74 and 0.71 , respectively, thereby demonstrating a very good diagnostic accuracy for predicting FMD in patients at low risk for cardiovascular disease. Although there were no significant differences between these outcome measures when comparing the AUC, the multiple logistic regression analysis showed that BS syndrome was a significant factor for FMD after adjusting for DLP and IGT, neither of which were significant in the model. Thus, the findings of this study suggest that BS syndrome might be more useful than IGT for discerning the presence of FMD in patients who are at low risk for cardiovascular events. 
TABLE 5: Areas under the curves for BS syndrome + IGT, BS syndrome, IGT, and DLP $(n=41)$.

\begin{tabular}{lccccc}
\hline Variables & Sensitivity & Specificity & AUC & SE & $95 \%$ CI \\
\hline BS syndrome + IGT & 0.70 & 0.79 & 0.80 & 0.07 & $0.65-0.94$ \\
BS syndrome & 0.70 & 0.79 & 0.74 & 0.07 & $0.60-0.89$ \\
IGT & 0.78 & 0.64 & 0.71 & 0.08 & $0.56-0.86$ \\
DLP & 0.44 & 0.79 & 0.62 & 0.07 & $0.47-0.76$ \\
\hline
\end{tabular}

BS: blood stasis; IGT: impaired glucose tolerance; DLP: dyslipidemia; AUC: area under the curve; CI: confidence interval; SE: standard error.
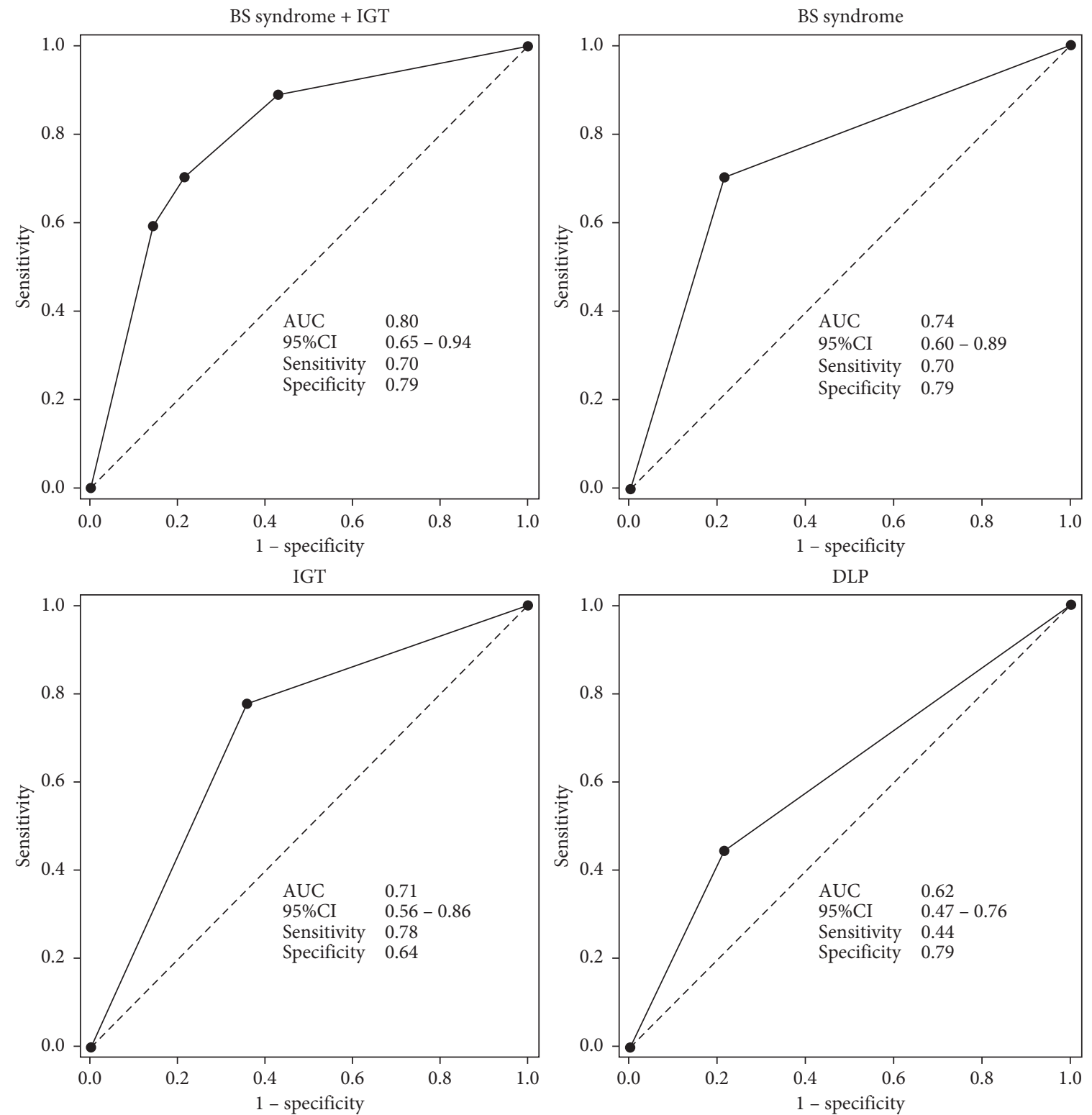

FIGURE 2: The diagnostic accuracy of blood stasis (BS) syndrome + impaired glucose tolerance (IGT), BS syndrome alone, IGT alone, and dyslipidemia (DLP) for predicting flow-mediated dilation (FMD). The receiver operating characteristic (ROC) curves depict that BS syndrome and IGT have good diagnostic utility for FMD, with BS syndrome + IGT having better diagnostic accuracy; DLP demonstrates poor diagnostic utility. In each graph, the solid diagonal line is the line of no discrimination (true area $=0.50$ ), and the optimal cutoff points are indicated on the curves. AUC: area under the curve; CI: confidence interval.

From the results of the multiple logistic regression analysis, the AUC of the combined parameter "BS syndrome + IGT" demonstrated better accuracy than that of BS syndrome or IGT alone. This suggests that BS syndrome combined with IGT may be a better discriminator of VED, as determined by FMD than by BS syndrome alone.

An association between BS syndrome and atherosclerotic changes based on the ABI [31] and the CAVI [5] has 
been reported; moreover, studies have shown a relationship between atherosclerotic changes and VED [27]. However, an association between BS syndrome and VED, as determined by FMD, has not been previously reported. In our study, contrary to the FMD results, the $\mathrm{ABI}$ and CAVI results were not significantly different between the groups.

The findings of this study should be considered in the light of three caveats. First, it should be considered that a lack of an association between BS syndrome and the physiological function tests (ABI and CAVI) may be explained by selecting patients who had a lower risk of cardiovascular events than those in previous studies [5]. Second, FMD was significantly lower for patients who had IGT with DLP, even though significant changes in ABI and CAVI were not found [32]. Results from previous studies and our study (shown in Table 2) are similar; therefore, a causal relationship of BS syndrome with VED can be considered plausible. Third, although sex-related differences in VED are thought to be related to age and hormonal status $[33,34]$, our findings indicate that there is no significant difference in FMD between male and female patients.

These results suggest that BS syndrome can represent a lower-grade abnormal functional change, and hence, arterial stiffness and stenosis represent morphological changes. Therefore, the diagnosis of BS syndrome in Kampo medicine can reflect early phases of atherosclerosis in patients with a relatively low risk of cardiovascular events.

In addition to our research, BS has been reported to be associated with atherosclerosis [35] and cardiovascular disease [36]. Both BS and VED are suspected to be associated with microangiopathy and atherosclerosis. Furthermore, VED is considered reversible [1]. Thus, early intervention and prevention are of critical importance. Improved FMD has been observed after the administration of hypotensive [37], hypolipidemic [38], and hypoglycemic agents [39], as well as with the implementation of exercise therapy [40], dietetic therapy [41], and smoking cessation [42]. Keishibukuryogan (Gui-zhi-fu-ling-wan in Chinese) has been shown to improve VED [43], which is considered to be the early phase of atherosclerosis. Zu San Li (ST36) and Nei Guan (PC6) in their randomized double-blind study showed an improvement in VED, as evaluated with FMD [44], using acupuncture.

Therefore, the findings of this study suggest that the diagnosis of BS syndrome may enable the implementation of lifestyle modifications, contributing to early intervention in the early stages of atherosclerosis. This will be of particular interest to medical staff in remote locations, disaster zones, and developing countries where patients may not be accessed to advanced medical care.

This study had several limitations. First, the ROC curves were difficult to interpret owing to the small sample size $(n=41)$. Second, although precedents exist in previous studies, the definition of cutoff values for FMD was arbitrary. Third, studies comparing arterial condition and blood stasis in this field are still few. Blood stasis in Kampo medicine includes the dysfunction of blood distribution not only in arteries but also in veins and capillaries. This study comprises only one of the components of blood stasis. Thus, validation with larger cohorts, methodological improvement, and strictly defined protocols are necessary for future studies.

\section{Conclusions}

Our study indicates that BS syndrome is a significant factor for VED, as determined by FMD. Furthermore, a superior diagnostic accuracy can be obtained by combining BS score with IGT. The findings of this study suggest that the BS syndrome in Kampo medicine can be a useful tool to detect early pathogenic stages of atherosclerosis.

\section{Data Availability}

The data used to support the findings of this study are available within the article and its supplementary materials.

\section{Conflicts of Interest}

The authors declare that there are no conflicts of interest regarding the publication of this article.

\section{Acknowledgments}

The authors are extremely grateful to Kobayashi Pharmaceutical Co., Ltd., and the Japan Agency for Medical Research and Development for their cooperation in this study. Kobayashi Pharmaceutical Co., Ltd., provided us with the flow-mediated dilation equipment (the equipment for vascular endothelial dysfunction).

\section{Supplementary Materials}

Table S1: physical findings. Table S2: background characteristics. Table S3: background characteristics and results of physiological function tests. Table S4: laboratory test results. (Supplementary Materials)

\section{References}

[1] W. A. Hsueh and M. J. Quiñones, "Role of endothelial dysfunction in insulin resistance," The American Journal of Cardiology, vol. 92, no. 4A, pp. 10J-17J, 2003.

[2] D. S. Celermajer, K. E. Sorensen, D. Georgakopoulos et al., "Cigarette smoking is associated with dose-related and potentially reversible impairment of endothelium-dependent dilation in healthy young adults," Circulation, vol. 88, no. 5, pp. 2149-2155, 1993.

[3] S. Yamamoto, N. Tsumura, T. Nakaguchi et al., "Principal component vector rotation of the tongue color spectrum to predict "Mibyou" (disease-oriented state)," International Journal of Computer Assisted Radiology and Surgery, vol. 6, no. 2, pp. 209-215, 2011.

[4] C. Matsumoto, T. Kojima, K. Ogawa et al., "A proteomic approach for the diagnosis of Oketsu'(blood stasis), a pathophysiologic concept of Japanese traditional (Kampo) medicine," Evidence-based Complementary and Alternative Medicine, vol. 5, no. 4, pp. 463-474, 2008.

[5] K. H. Cho, K. P. Kim, B. C. Woo et al., "Relationship between blood stasis syndrome score and cardioankle vascular index in 
stroke patients," Evidence-based Complementary and Alternative Medicine, vol. 2012, Article ID 696983, 7 pages, 2012.

[6] K. Terasawa, H. Shinoda, A. Imadaya et al., "The presentation of diagnostic criteria for "Yu-Xie" (stagnated blood) conformation," International Journal of Oriental Medicine, vol. 14, pp. 194-213, 1989.

[7] K. Terasawa, T. Itoh, Y. Morimoto, Y. Hiyama, and H. Tosa, "The characteristics of the microcirculation of bulbar conjunctiva in "oketsu" syndrome," Journal of Medical and Pharmaceutical Society for WAKAN-YAKU, vol. 5, pp. 200205, 1988.

[8] T. Itoh, K. Terasawa, K. Kohta et al., "Effects of Keishibukuryo-gan and Trapidil on the microcirculation in patients with cerebro-spinal vascular disease," Journal of Medical and Pharmaceutical Society for WAKAN-YAKU, vol. 9, pp. 40-46, 1992.

[9] H. Hikiami, K. Kohta, N. Sekiya et al., "Erythrocyte deformability in "oketsu" syndrome and its relations to erythrocyte viscoelasticity," Journal of Traditional Medicines, vol. 13, pp. 156-164, 1996.

[10] N. Shibahara, Y. Shimada, N. Sekiya et al., "Effect of Keishibukuryo-gan on autonomic nervous activity," Journal of Traditional Medicines, vol. 20, pp. 62-67, 2003.

[11] N. Shibahara, “"Correlation between "oketsu” syndrome and autonomic nervous activity," Journal of Traditional Medicines, vol. 21, no. 1, pp. 1-6, 2004.

[12] H. Hikiami, K. Nozaki, Y. Nagata et al., "Biomarkers of endothelial dysfunction are elevated in patients with rheumatoid arthritis with oketsu (blood stasis)," Journal of Traditional Medicines, vol. 25, no. 4, pp. 103-107, 2008.

[13] L. Vitiello, I. Spoletini, S. Gorini et al., "Microvascular inflammation in atherosclerosis," IJC Metabolic \& Endocrine, vol. 3, pp. 1-7, 2014.

[14] T. Tomita, A. Hirayama, H. Matsui, and K. Aoyagi, "Effect of Keishibukuryogan, a Japanese traditional Kampo prescription, on improvement of microcirculation and oketsu and induction of endothelial nitric oxide: a live imaging study," Evidence-based Complementary and Alternative Medicine, vol. 2017, Article ID 3620130, 7 pages, 2017.

[15] Japanese Circulation Society, Guidelines for Non-invasive Vascular Function Test, Japanese Circulation Society, Tokyo, Japan, 2013pp. 3-112, in Japanese, https://www.j-circ.or.jp/ cms/wp-content/uploads/2020/02/JCS2013_yamashina_h.pdf.

[16] R. J. Esper, R. A. Nordaby, J. O. Vilariño, A. Paragano, J. L. Cacharrón, and R. A. Machado, "Endothelial dysfunction: a comprehensive appraisal," Cardiovascular Diabetology, vol. 5 , no. 1 , p. 4,2006 .

[17] A. Umeda, T. Kato, T. Yamane et al., "Does smoking cessation with varenicline worsen vascular endothelial function?" $B M J$ Open, vol. 3, no. 6, Article ID e003052, 2013.

[18] K. Takeno, T. Mita, S. Nakayama et al., "Masked hypertension, endothelial dysfunction, and arterial stiffness in type 2 diabetes mellitus: a pilot study," American Journal of Hypertension, vol. 25, no. 2, pp. 165-170, 2012.

[19] H. Tomiyama, Y. Higashi, B. Takase et al., "Relationships among hyperuricemia, metabolic syndrome, and endothelial function," American Journal of Hypertension, vol. 24, no. 7, pp. 770-774, 2011.

[20] M. Kinouchi, K.-i. Aihara, Y. Fujinaka et al., "Diabetic conditions differentially affect the endothelial function, arterial stiffness and carotid atherosclerosis," Journal of Atherosclerosis and Thrombosis, vol. 21, no. 5, pp. 486-500, 2014.
[21] J. W. Butany, S. Verma, R. L. Leask, B. Mohsen, and S. L. Asa, "Genetic abnormalities of the endothelium," Microscopy Research and Technique, vol. 60, no. 1, pp. 30-37, 2003.

[22] H. Shimokawa, "Primary endothelial dysfunction: Atherosclerosis," Journal of Molecular and Cellular Cardiology, vol. 31, no. 1, pp. 23-37, 1999.

[23] C. G. Schalkwijk and C. D. A. Stehouwer, "Vascular complications in diabetes mellitus: the role of endothelial dysfunction," Clinical Science, vol. 109, no. 2, pp. 143-159, 2005.

[24] M. Charakida, S. Masi, T. F. Lüscher, J. J. P. Kastelein, and J. E. Deanfield, "Assessment of atherosclerosis: the role of flow-mediated dilatation," European Heart Journal, vol. 31, no. 23, pp. 2854-2861, 2010.

[25] D. H. J. Thijssen, M. A. Black, K. E. Pyke et al., "Assessment of flow-mediated dilation in humans: a methodological and physiological guideline," American Journal of PhysiologyHeart and Circulatory Physiology, vol. 300, no. 1, pp. H2-H12, 2011.

[26] T. Oji, T. Namiki, T. Nakaguchi et al., "Study of factors involved in tongue color diagnosis by Kampo medical practitioners using the Farnsworth-Munsell 100 hue test and tongue color images," Evidence-based Complementary and Alternative Medicine, vol. 2014, Article ID 783102, 9 pages, 2014.

[27] A. D. Protogerou, E. Zampeli, K. Fragiadaki, K. Stamatelopoulos, C. Papamichael, and P. P. Sfikakis, “A pilot study of endothelial dysfunction and aortic stiffness after interleukin-6 receptor inhibition in rheumatoid arthritis," Atherosclerosis, vol. 219, no. 2, pp. 734-736, 2011.

[28] T. Furumoto, S. Fujii, N. Saito, T. Mikami, and A. Kitabatake, "Relationships between brachial artery flow mediated dilation and carotid artery intima-media thickness in patients with suspected coronary artery disease," Japanese Heart Journal, vol. 43, no. 2, pp. 117-125, 2002.

[29] B. C. K. Choi, "Slopes of a receiver operating characteristic curve and likelihood ratios for a diagnostic test," American Journal of Epidemiology, vol. 148, no. 11, pp. 1127-1132, 1998.

[30] K. H. Zou, A. J. O’Malley, and L. Mauri, "Receiver-operating characteristic analysis for evaluating diagnostic tests and predictive models," Circulation, vol. 115, no. 5, pp. 654-657, 2007.

[31] K. C. Huang, C. C. Chen, Y. C. Su et al., "The relationship between stasis-stagnation constitution and peripheral arterial disease in patients with type 2 diabetes," Evidence-based Complementary and Alternative Medicine, vol. 2014, Article ID 903798, 6 pages, 2014.

[32] K. Tsuchiya, C. Nakayama, F. Iwashima et al., "Advanced endothelial dysfunction in diabetic patients with multiple risk factors; importance of insulin resistance," Journal of Atherosclerosis and Thrombosis, vol. 14, no. 6, pp. 303-309, 2007.

[33] D. S. Celermajer, K. E. Sorensen, D. J. Spiegelhalter et al., "Aging is associated with endothelial dysfunction in healthy men years before the age-related decline in women," Journal of the American College of Cardiology, vol. 24, no. 2, pp. 471-476, 1994.

[34] C. Cao, J. Hu, Y. Dong et al., "Gender differences in the risk factors for endothelial dysfunction in Chinese hypertensive patients: homocysteine is an independent risk factor in females," PLoS One, vol. 10, no. 2, Article ID e0118686, 2015.

[35] Z. Liu, H. Yang, M. Zhang, J. Cai, and Z. Huang, "The interaction effect between blood stasis constitution and atherosclerotic factors on cognitive impairment in elderly people," Evidence-based Complementary and Alternative Medicine, vol. 2018, Article ID 8914090, 8 pages, 2018. 
[36] Y. Wang, C. Li, H. Chang et al., "Metabolomic profiling reveals distinct patterns of tricarboxylic acid disorders in blood stasis syndrome associated with coronary heart disease," Chinese Journal of Integrative Medicine, vol. 22, no. 8, pp. 597-604, 2016.

[37] A. R. Zankl, B. Ivandic, M. Andrassy et al., "Telmisartan improves absolute walking distance and endothelial function in patients with peripheral artery disease," Clinical Research in Cardiology, vol. 99, no. 12, pp. 787-794, 2010.

[38] O. Timár, Z. Szekanecz, G. Kerekes et al., "Rosuvastatin improves impaired endothelial function, lowers high sensitivity CRP, complement and immuncomplex production in patients with systemic sclerosis - a prospective case-series study," Arthritis Research \& Therapy, vol. 15, no. 5, p. R105, 2013.

[39] Y. Kubota, M. Miyamoto, G. Takagi et al., "The dipeptidyl peptidase-4 inhibitor sitagliptin improves vascular endothelial function in type 2 diabetes," Journal of Korean Medical Science, vol. 27, no. 11, pp. 1364-1370, 2012.

[40] M. Igaki, H. Honda, M. Komatsu, and M. Sakaue, "Effect of exercise therapy on flow-mediated dilation in type 2 diabetes mellitus and non-diabetes patients," Journal of Allied Health Sciences, vol. 8, no. 1, pp. 23-29, 2017.

[41] K. M. Dickinson, J. B. Keogh, and P. M. Clifton, "Effects of a low-salt diet on flow-mediated dilatation in humans," The American Journal of Clinical Nutrition, vol. 89, no. 2, pp. 485-490, 2009.

[42] K. Yufu, N. Takahashi, M. Hara, T. Saikawa, and H. Yoshimatsu, "Measurement of the brachial-ankle pulse wave velocity and flow-mediated dilatation in young, healthy smokers," Hypertension Research, vol. 30, no. 7, pp. 607-612, 2007.

[43] Y. Nagata, H. Goto, H. Hikiami et al., "Effect of Keishibukuryogan on endothelial function in patients with at least one component of the diagnostic criteria for metabolic syndrome: a controlled clinical trial with crossover design," Evidencebased Complementary and Alternative Medicine, vol. 2012, Article ID 359282, 10 pages, 2012.

[44] J.-M. Park, A.-S. Shin, S.-U. Park, I.-S. Sohn, W.-S. Jung, and S.-K. Moon, "The acute effect of acupuncture on endothelial dysfunction in patients with hypertension: a pilot, randomized, double-blind, placebo-controlled crossover trial," The Journal of Alternative and Complementary Medicine, vol. 16, no. 8, pp. 883-888, 2010. 